\title{
Modeling control over large space structure on geostationary orbit
}

\author{
V.V. Salmin ${ }^{1}$, A.S. Chetverikov ${ }^{1}$, K.V. Peresypkin ${ }^{1}$, I.S. Tkachenko ${ }^{1}$

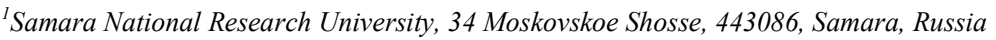

\begin{abstract}
The paper considers the problem of control over a large space structure (LSS) control at a given station on a geostationary orbit (GSO). An observation spacecraft with diffractive optical elements (DOE) is taken as an example of a large space structure. Various perturbing factors influence the motion of a LSS along GSO, most notably solar radiation pressure. Two problems are considered: control over the motion of the center of mass, and control of the motion in relation to the center of mass. The paper gives the results of modeling the process of LSS control, based on developed control algorithms.
\end{abstract}

Keywords: modeling; large space structure; low thrust; terminal control; geostationary orbit; solar radiation pressure; controlling torque

\section{Introduction}

The paper considers an observation spacecraft with diffractive optical elements (DOE). The optical scheme is taken as in DAPRA's MOIRE project [1]. This observation spacecraft can be defined as a large space structure: DOE assembly diameter is 10 meters, and the distance from the optical elements to the body of the spacecraft is 60 meters. The design of the frame that connects DOE to the body is discussed in detail in [2].

Further work on the project resulted in development of a hood that prevents any light that doesn't come from the observed object from reaching the optical elements. The hood is a system of surfaces that are so located that they shield the optical element in the body of the spacecraft from any rays that do not come from the observed object, and also to shield the rear surface of DOEs. The observation spacecraft with the hood is shown in Figure 1.

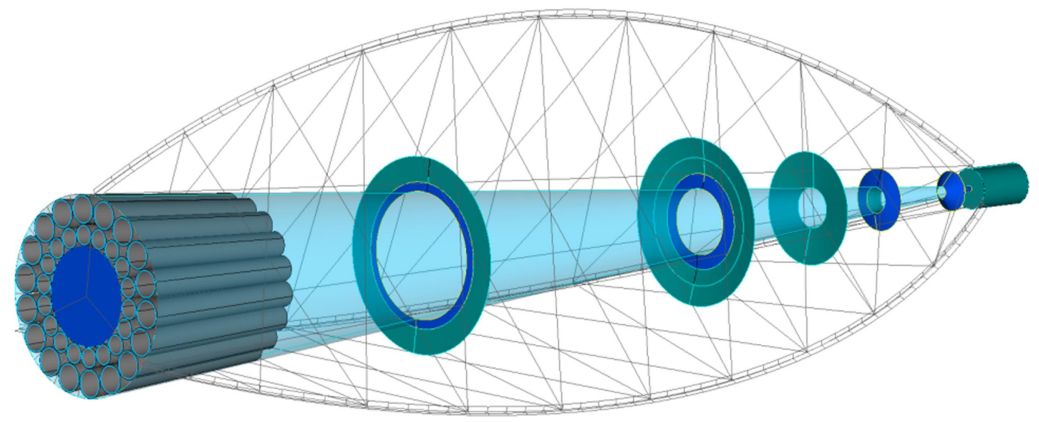

Fig. 1. Observation spacecraft with envelope- and ring-shaped hoods for diffractiive optical elements.

The orbital movement of a space structure with such mass and dimensions will be highly influenced by various perturbing factors, particularly by torque and momentum created by solar radiation pressure. In addition, as the spacecraft is moving along its orbit, it will experience quite noticeable perturbing accelerations from the gravity fields of the Sun and the Moon. For these reasons, keeping the orbital structure at the desired station on GSO requires constant trajectory correction. In addition, attitude control is necessary to keep the roll axis of the optical structure under consideration pointed to the Earth at all times.

\section{Modeling the algorithms of terminal control over the motion of the LSS's center of mass.}

\subsection{Setting the goals of control.}

The goal of control is to keep the final state deflection vector $\Delta X_{K}$ within the allowable area $G_{Д}$. The adjustment maneuver is performed by applying transversal torque with the help of a low thrust electric propulsion (EP) unit. The transversal torque creates acceleration $a_{T}$ along the transversal.

This problem is stated as an optimal control problem with the functional

$$
I=\Delta x_{K}^{T} \Lambda \Delta x_{K} \rightarrow \min ,
$$

where $\Lambda$ is the constant coefficients matrix.

The control is structured as a sequence of lengths of powered and unpowered flight $u=\left\{\tau_{1} \ldots \tau_{i}, t_{\Pi 1} \ldots t_{\Pi i}\right\}^{T}$

Deviation of the orbit's semi-major axis $\Delta A$ of the orbit is equivalent to the deviation of the orbit time of the spacecraft $\Delta T=T$ $-T_{3}$. Here the orbit time of the spacecraft on the geostationary orbit equals star day $T_{3}=86164.09 \mathrm{c}$. In addition, the spacecraft's position on the orbit is determined by longitude $\lambda$, which differs from the required value of the station longitude $\lambda_{P}$ by $\Delta \lambda=\lambda-$ $\lambda_{P}$ 


\subsection{Solving the terminal control problem with a multistep algorithm.}

The terminal control problem is solved with the help of a multistep algorithm with adjustment of control parameters. Let the control law be set by a sequence of thrust lengths, which is taken as decreasing, and defined by the expression [3]:

$$
\tau_{i}=a \cdot\left[1-\left(\frac{i-1}{n}\right)^{b}\right],
$$

where $i, n$ are the number of the adjustment and the total number of adjustments respectively; $a, b$ are the parameters that characterize the law of decreasing lengths of thrusts.

Then the problem of determining the optimal control law is reduced to a two-parameter optimization problem, which is stated in the following way: for the set initial values of the orbital elements, transversal acceleration $a_{T}$, number of corrections $n$, lengths of unpowered flight $t_{\Pi}$ one must find such parameters $a$ and $b$ that would ensure the minimum of the functional (see formula (1)).

A peculiar feature of the algorithm presented in this paper is that the control parameters $a$ and $b$ are found as the result of minimization of the functional (1) and at the same time, for better precision, the relation of the functional to the parameter $a$ is approximated by the least squares method. When the control is adjusted (during motion modeling with allowances for perturbations) at every unpowered leg the number of steps $n$ is also adjusted.

A series of calculations of the control laws for transfer of an EP-powered spacecraft into a given station by longitude and orbit time have been carried out. The delta $\mathrm{V}$ expense, depending on the initial value of deviation by orbit time $\left(\Delta T_{0}=\right.$ $300 \ldots 1000 \mathrm{c}$ ) ranges from 4 to $14 \mathrm{~m} / \mathrm{s}$.

\subsection{Results of modeling terminal control with the help of a multi-step algorithm.}

Tables 1 and 2, and figures 3 and 4 give sample calculations of control parameters (see Formula (2)) for a given station on GSO, for two cases, with and without adjustment. Figure 3 represents phase trajectory of a spacecraft transfer to a given GSO station without adjusting control parameters. The maneuver was modeled with the help of equations of motion in equinoctial elements.

Figure 2 shows the regions of deviation of the final orbit time and longitude divergence values for the EP-powered observational spacecraft transfer control law, without adjustment of control parameters, and with stepwise adjustment at every stage of the transfer. The process was modeled with allowances for perturbing accelerations from the gravitational fields of the Sun, the Moon, the Earth, and solar radiation pressure.

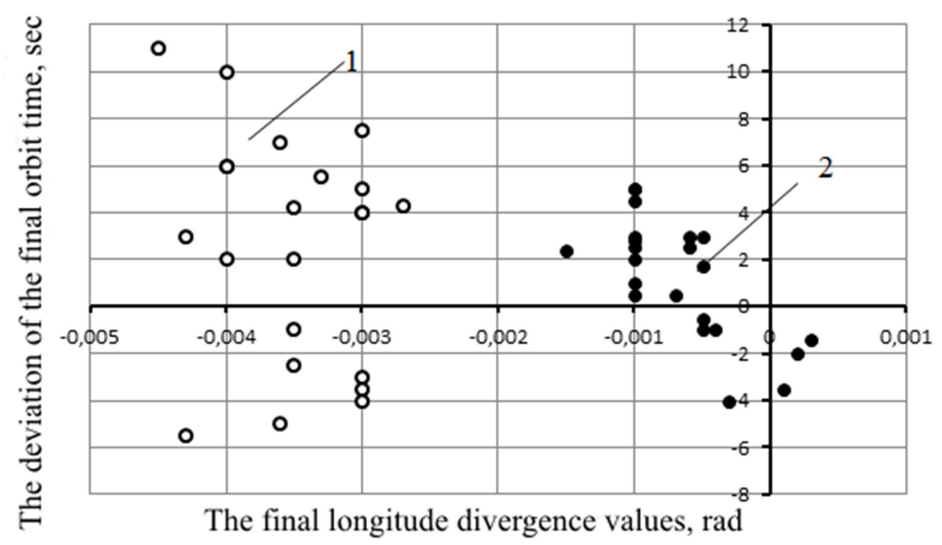

Fig. 2. The area of the final values of $\Delta \mathrm{T}$ and $\Delta \lambda 1$ ) without, and 2) with adjustment of control parameters.

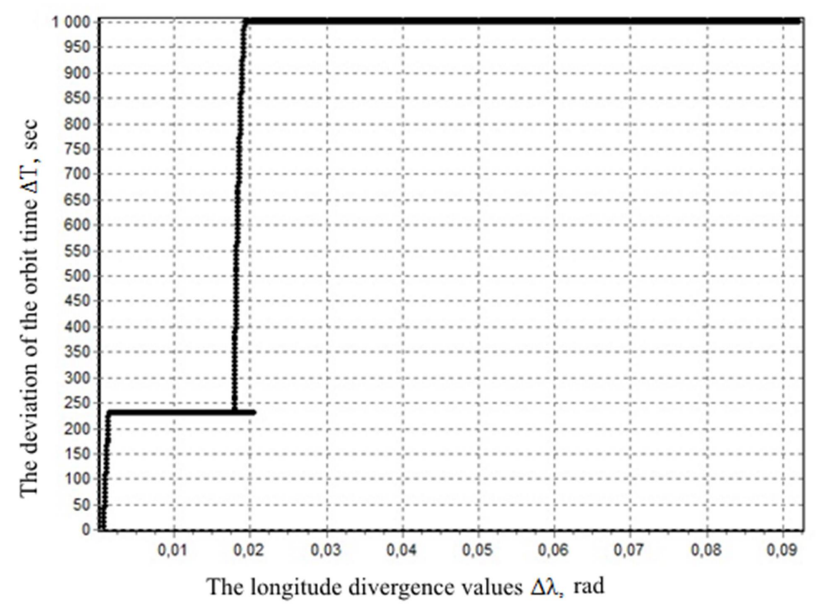

Fig. 3. Phase trajectories of the transfer of EP-powered spacecraft to a given GSO station. 


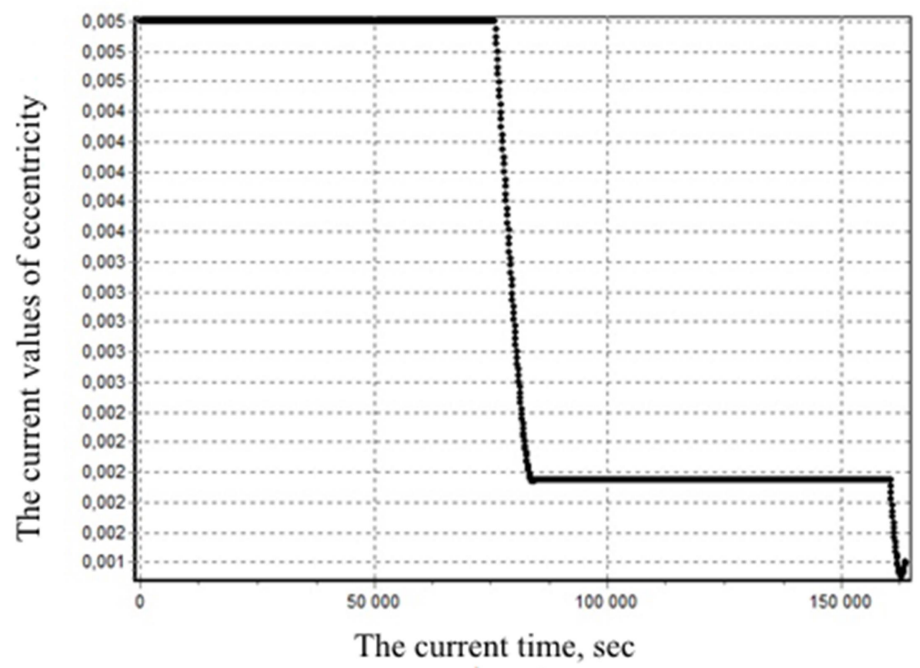

Fig. 4. Changes in eccentricity during transfer of an EP-powered spacecraft to given station at GSO.

Table 1. Control parameters for transfer to a given station at GSO $\left(\Delta T_{0}=1000 \mathrm{c}, a_{T}=0,001 \mathrm{M} / \mathrm{c}^{2}, t_{\Pi}=76000 \mathrm{c}, \Delta \lambda_{0}=0,092 \mathrm{rad}\right.$,

\begin{tabular}{|c|c|c|c|c|c|c|c|}
\hline \multicolumn{8}{|c|}{$\left.\lambda_{\mathrm{P}}=75,1^{0}\right)$} \\
\hline № & $n$ & $a$ & $b$ & $V_{X K}, \mathrm{~km} / \mathrm{s}$ & $\Delta T_{K}, \mathrm{sec}$ & $\Delta \lambda_{\mathrm{K}}, \mathrm{rad}$ & $\Delta e_{K}$ \\
\hline \multicolumn{8}{|c|}{ Without refinement of control parameters } \\
\hline 1 & 2 & 8460,5 & 0,7 & 0,012 & 6 & $-0,0025$ & 0,0018 \\
\hline \multicolumn{8}{|c|}{ With refinement of control parameters. } \\
\hline 1 & 2 & 8441,5 & 0,7 & & & & \\
\hline 2 & 2 & 3105,7 & 0,1 & 0,012 & 2 & $-0,0005$ & 0,0010 \\
\hline 3 & 1 & 76,6 & 2,2 & & & & \\
\hline
\end{tabular}

Table 2. Lengths of powered flight legs $\left(\Delta T_{0}=1000 \mathrm{c}, a_{0}=0,001 \mathrm{M} / \mathrm{c}^{2}, t_{\Pi}=76000 \mathrm{c}, \Delta \lambda_{0}=0,092 \mathrm{rad}\right)$

\begin{tabular}{lcl}
\hline$\tau_{1}, \mathrm{c}$ & $\tau_{3}, \mathrm{c}$ \\
\hline \multicolumn{2}{l}{ Without adjustment of control parameters } \\
\hline $\mathbf{8 4 6 0 , 5}$ & - \\
\hline \multicolumn{2}{l}{ With adjustment of control parameters } \\
\hline 8441,5 & 76,6 \\
\hline
\end{tabular}

\section{Modeling rotation of a large space structure about its center of mass}

\subsection{Setting of the problem}

Accelerations on the orbit are determined by maneuvers of positioning the spacecraft to point at the object of observation. The values of the turn angles depend not only on the mutual position of observed objects, but also on perturbing factors that influence the spacecraft's angular position in relation to the Earth. One such factor is the rotation of the spacecraft on its orbit: if the spacecraft does not itself rotate in relation to the inertial system of coordinates, then as it moves along the orbit the optical axis will turn in relation to the Earth. This factor can be negated by ensuring the spacecraft's rotation about its axis at the same rate as the spacecraft's orbit time.

However, other perturbations will alter the angular spin rate of the spacecraft, shifting its optical axis away from the Earth. On a geostationary orbit, with the dimensions of the spacecraft under consideration, the biggest perturbing factor will be solar radiation pressure. Most of the spacecraft's mass is located in its body; in fact, the center of mass is only $3.25 \mathrm{~m}$. from the body. Yet, the main center of solar radiation pressure, according to Figure 1, will be in the neighborhood of the diffractive optical elements, which will lead to production of a substantial moment of rotational force.

To estimate the value of the solar radiation induced turning torque, let us assume that there is no reflection and the solar radiation is completely absorbed by the spacecraft. Then the direction of the solar radiation pressure will coincide with the direction of the sunlight. The area of the radiation beam that falls on the spacecraft and the distance from center of mass to center of pressure depend on the angle of exposure. The value of the rotating torque will be found as the multiplication of these values, and is represented in Figure 5. 


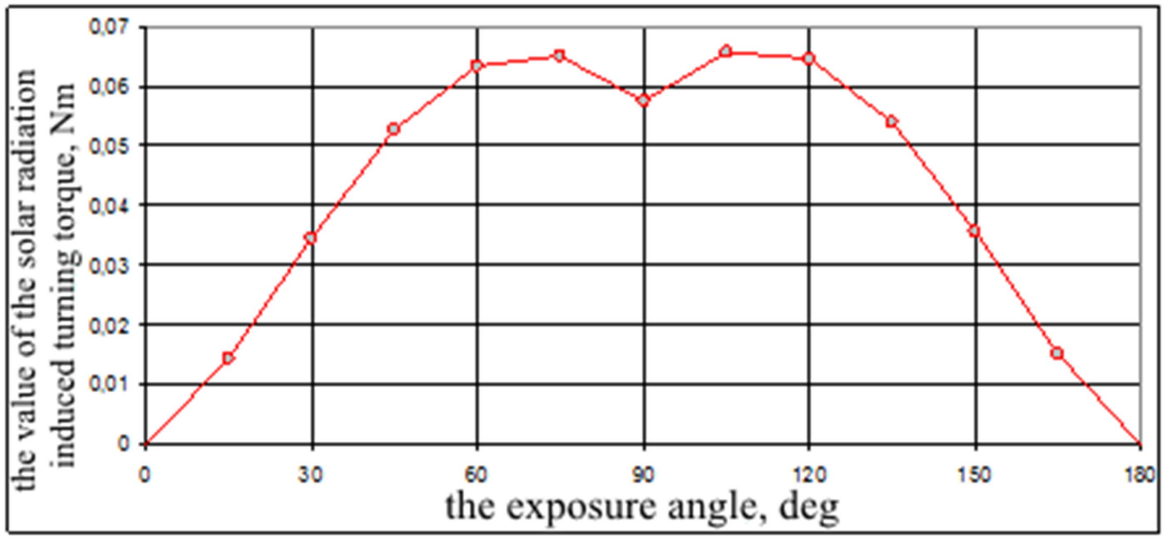

Fig. 5. Relation of rotational torque to exposure angle.

For the structure under consideration, at the maximum torque value the spacecraft will turn by $0,67^{\circ}$ in 10 minutes. This is a significant perturbation of motion, and without correction the spacecraft will quite soon turn away from the Earth so much that imaging will be impossible.

Frequent correction is necessary to keep the spacecraft in proper position for imaging. During correction, the controlling impulse will cause vibrations in the spacecraft's frame. Movement of diffractive optical elements in relation to the body creates problems for the spacecraft's optical system. Therefore, it is necessary to select such type of controlling impact that would ensure that the vibrations are damped soon enough not to interfere with the mission of the spacecraft. The control problem for such a large space structure must be solved with allowances for elasticity in its design. Turning of the spacecraft is modeled with the help of the finite elements method.

During the turn, the spacecraft rotates in the inertial system of coordinates, and elastic vibrations occur in its frame. The amplitudes of the vibrations are expected to be small, i.e., not powerful enough to change the elastic and inertial properties of the spacecraft's structure. If the spacecraft is considered in a system of coordinates tied to it, then geometrical non-linearity is absent. Modeling is performed in the inertial system of coordinates for convenience of setting the boundary conditions and analysis of the results. In this case, when the finite-element model of the design turns, one must re-calculate the matrix of masses, dampening, and rigidity for the new orientation of finite elements in space. However, the angle of the turn is small, and the related changes in the matrixes are minor. Let us neglect the impact of the turn of the spacecraft on the matrix coefficients and consider the system as linear. Let us now apply the MSC Nastran linear transition analysis. This analysis performs numerical integration of the main dynamic equation in time [4]:

$$
[M] \cdot\{u\}+[C] \cdot\{u\}+[K] \cdot\{u\}=\{P(t)\},
$$

where $\{P(t)\}$ is the nodal forces vector; $\mathrm{j}$ is the number of the integration step; $\{u\}$ is the vector of the nodal movement of the model; $\{u\}$ is the model's nodal velocity vector; $\{u\}$ is the model's nodal accelerations vector; $[C]$ is the dampening matrix. The velocities and accelerations are expressed through motion via central-differential approach:

$$
\{u\}_{j}=\frac{\{u\}_{j+1}-\{u\}_{j-1}}{2 \cdot \Delta t} ;\{u\}_{j}=\frac{\{u\}_{j+1}-\{u\}_{j-1}}{2 \cdot \Delta t}
$$

where $\Delta t$ is the step of integration in time. Then, with averaging the nodal forces vector for three neighboring steps in time, the system (see (Formula3)) is transformed to the following view:

$$
\left[A_{1}\right] \cdot\{u\}_{j+1}=\left[A_{2}\right] \cdot\{u\}_{j}+\left[A_{3}\right] \cdot\{u\}_{j-1}+\left[A_{4}(t)\right]
$$

Calculation of motion with the help of a system of linear equations (see (Formula 5)) was performed for initial conditions of $\{u\}_{j}=0$ and $\{u\}_{j-1}=0$, which corresponds to immobile spacecraft at the initial moment in time.

\subsection{Modeling results}

Two laws of controlling torque change were considered:

1) Two consequent "square" torque impulses in different directions. This corresponds to minimal values of the controlling torque at a given time and angle of turn;

2) Two consequent "smoothened" torque impulses in different directions. The shape of the smoothened impulses is taken as in the formula (6). Smooth change of controlling torque in this case is meant to decrease the amplitude of vibrations of the spacecraft's frame after the turn is completed, as compared to "square" torque impulses. 


$$
M_{\text {cont }}(t)=\left\{\begin{array}{cc}
0,5 \cdot M_{\max } \cdot\left(1-\cos \left(4 \pi \frac{t}{T_{k}}\right)\right), & \text { with } 0 \leq t<T_{k} / 2, \\
-0,5 \cdot M_{\max } \cdot\left(1-\cos \left(4 \pi \frac{t}{T_{k}}\right)\right), & \text { with } T_{k} / 2 \leq t \leq T_{k},
\end{array}\right.
$$

Table 3 gives the parameters of turns for these two torque control laws. The view of the obtained relation of rotation of the DOEs about the body of the spacecraft is represented in Figure 6.

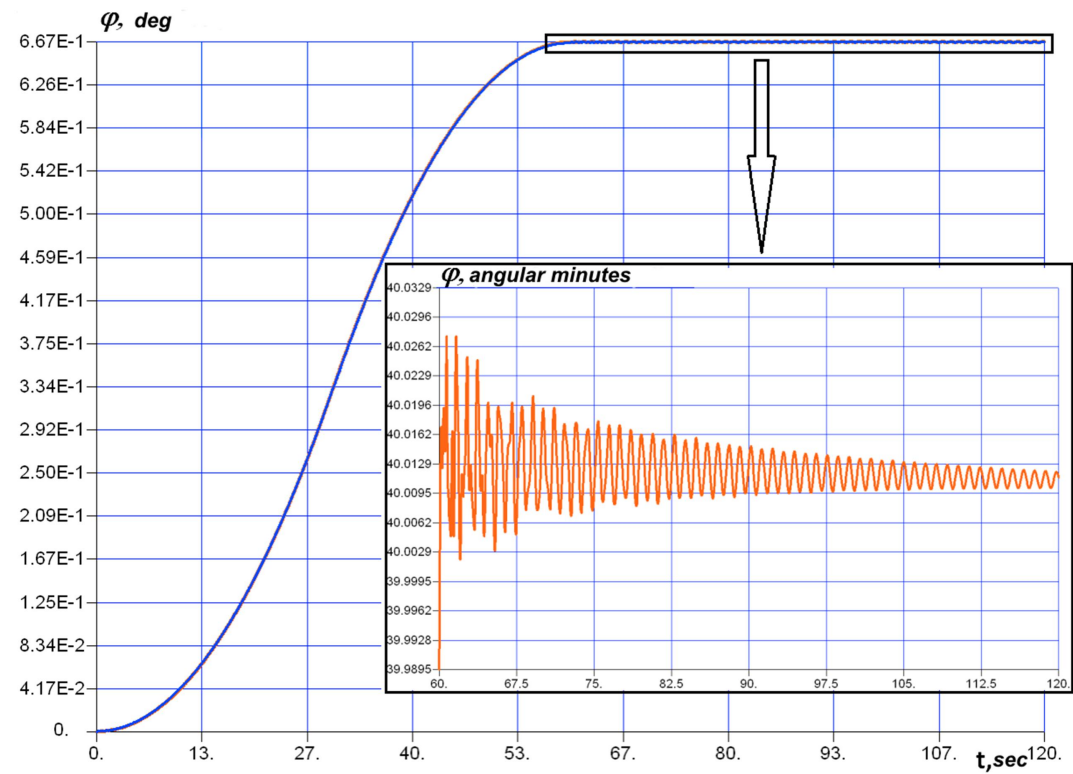

Fig. 6. The angle of the turn of the spacecraft for "square" controlling torque impulses.

Table 3. Parameters of the turn.

Controlling torque change law

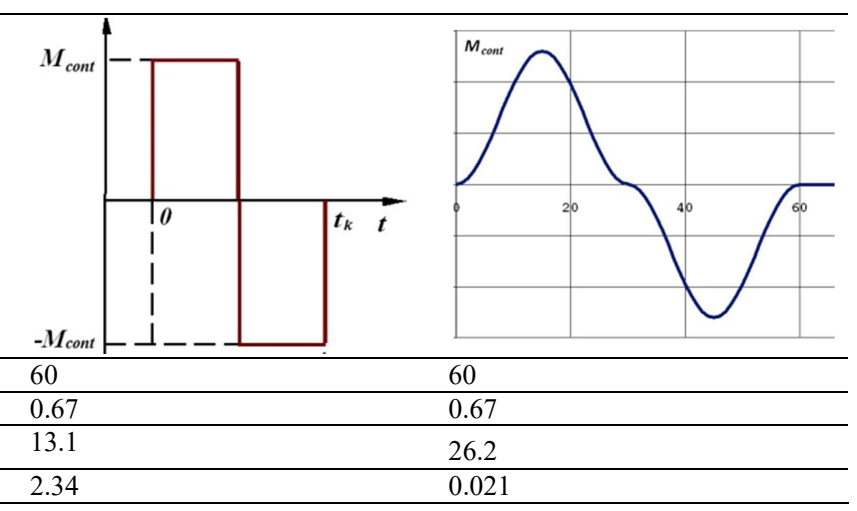

As seen from Table 3, adoption of the "smoothened" torque control law reduced the amplitude of vibration by order of two. The maximum controlling torque value, however, increased twofold, which would require the spacecraft to be equipped with more powerful control thrusters. The results lead to the conclusion that the problem of reducing vibrations in the spacecraft's optical system elements can be efficiently resolved in this case by the choice of the type of controlling impact.

\section{Conclusion}

The process of controlling a large-dimensional structure shown in Fig. 1 was modeled, using the specially developed multistep terminal control algorithm that allows to account for perturbing accelerations. Adjustment of control parameters during the correction maneuver allows to reduce the final deviations of the orbital parameters (see Fig. 2). However, the considered multistep algorithm has a disadvantage. To achieve the required eccentricity value at the end of the transfer to the given station point, the lengths of the passive parts of the transfer has to be hand-picked, which makes the search for the solution of the problem more complicated and not always successful.

Dynamic calculation and modeling of a turn of an observation spacecraft were carried out for two variants of torque control law: with "square" and "smoothened" change of the controlling torque.

In the first instance, which ensures the quickest turn, the amplitudes of vibration in the optical elements and the body of the spacecraft are $\sim 0,039$ minutes of angle immediately after the turn, and $\sim 2,9 \mathrm{E}-4$ minutes of angle 60 seconds after the end of the turn. In the second instance the amplitudes of vibration in the optical elements and the body of the spacecraft are $\sim 3,5 \mathrm{E}-4$ ' 
minutes of angle immediately after the turn, and $\sim 1,0 \mathrm{E}-4$ ' minutes of angle 60 seconds after. The use of the "smoothened" torque control law increases the maximum value of controlling torque by two (to $26 \mathrm{H} \cdot \mathrm{m}$ for the turn under consideration), but decreases the vibrations produced during the maneuver by order of two.

\section{Acknowledgements}

The research was carried out with financing within the framework of state order № 9.1004.2014/K.

\section{References}

[1] Atcheson P, Stewart C, Domber J, Whiteaker K, Cole J, Spuhler P, Seltzer A, Smith L . MOIRE - Initial demonstration of a transmissive diffractive membrane optic for large lightweight optical telescopes. Proceedings of SPIE - The International Society for Optical Engineering 2012 ; 8442 : 844221.

[2] Salmin VV, Karpeev SV, Peresypkin KV, Chetverikov AS, Tkachenko IS. Feasibility study and modeling of components for an informational space system based on a large diffractive membrane. CEUR Workshop Proceedings 2016; 1638: 132-148.

[3] Chernyavsky GM, Bartenev BA, Malyshev V A. Controlling the orbit of a geostationary satellite. Moscow: Mashinostroyenie 1984; 144 p. (in Russian)

[4] MSC.Nastran. Reference Manual: The Official Web Site of the Corporation, 2004. URL: https://simcompanion.mscsoftware.com/resources/ sites/MSC/content/meta/DOCUMENTATION/9000/DOC9188/ secure/refman.pdf. 